\title{
Alle paar Jahre wieder - Dynamik und Steuerungsversuche des EU-Erweiterungsprozesses
}

\author{
Barbara Lippert*
}

Unter „Erweiterung“ ist die Aufnahme neuer Mitglieder gemäß den einschlägigen Arti-

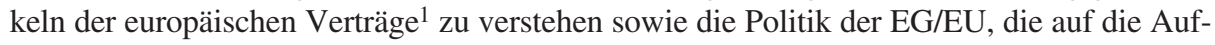
nahme neuer Mitglieder abzielt. Erweiterung ist jedoch mehrdimensional, weil sie das gesamte EU-System in Bezug auf seine policies, politics und polity betrifft. So hat der Beitritt eines neuen Mitglieds in jedem Fall wegen der notwendigen Anpassung der Verträge primärrechtliche Folgen, es verändern sich Konstellationen im Hinblick auf die Europastrategien der EU-Akteure und es werden Politiken der Gemeinschaft beeinflusst. Manches davon findet antizipierend vor, anderes nach dem Beitrittstermin statt. Ob diese internen Entwicklungen jeweils kausal der Erweiterung zuzuschreiben sind, oder sie vorwiegend rhetorisch als Schwungrad für Veränderungen genutzt werden, ist im Einzelfall zu prüfen und schwierig zu belegen. ${ }^{2}$

In einem allgemeinen Verständnis kann ,Erweiterung“ als Prozess horizontaler Institutionalisierung von Werten, Normen und Regeln der EG/EU definiert und der vertikalen Institutionalisierung, also der Vertiefung, gegenüber gestellt werden. ${ }^{3}$ Es geht um die Ausdehnung der geografischen Reichweite und des Besitzstandes der Gemeinschaften auf Drittstaaten. In dieser Definition wird Erweiterung als ein Kontinuum, nicht als das Ereignis der Aufnahme verstanden und auf diese Weise problematisiert, dass auf Seiten des Bewerbers beziehungsweise neuen Mitglieds wie auch des EU-Systems Anpassungen im Vorlauf und im Nachgang zum Beitritt gefordert und zu beobachten sind. Die Erweiterung der Gemeinschaften wird häufig in deterministischer Weise als geradezu zwangsläufig ablaufender Prozess dargestellt, sei es reaktiv-fatalistisch oder strategisch als Erfüllung eines Plans der Nachkriegsgeschichte zur Vollendung der Einigung Europas, anders ausgedrückt, als ,natürliche Lösung“ für die ,unnatürliche Teilung Europas“.4

Was also ist - im Lichte der Etappen des Integrationsprozesses seit den Römischen Verträgen - überhaupt erklärungsbedürftig an der Erweiterung der EG/EU? Zwei Fragen sollen eingehender behandelt werden: Zum einen ist die Dynamik der Erweiterung, also das schrittweise Anwachsen von sechs auf einstweilen 27 Mitglieder zu erklären. Zweitens sind

* Dr. Barbara Lippert, Stellvertretende Direktorin des Instituts für Europäische Politik, Berlin; Lehrbeauftragte an der Humboldt Universität zu Berlin.

Der Aufsatz ist im Rahmen des IEP-Schwerpunktprogramms „Dialog Europa der Otto Wolff-Stiftung“ entstanden und wurde in Grundzügen auf der wissenschaftlichen Konferenz „,50 Jahre Römische Verträge“vorgetragen. Die Konferenz wurde vom Institut für Europäische Politik in Zusammenarbeit mit dem Arbeitskreis Europäische Integration und dem Centre International de Formation Européenne in Berlin durchgeführt.

1 Vgl. Art. 98 EGKS-Vertrag vom 18.4.1951; Art. 237 EWG-Vertrag vom 25.3.1957; Art. 205 des EuratomVertrags vom 25.3.1957; Art. 49 EU-Vertrag vom 7.2.1992 in der Fassung vom 26.2.2001 sowie die jeweiligen Präambeln.

2 Vgl. Barbara Lippert: Glanzloser Arbeitserfolg von epochaler Bedeutung: eine Bilanz der EU-Erweiterungspolitik 1989-2004, in: Barbara Lippert (Hrsg.): Bilanz und Folgeprobleme der EU-Erweiterung, Baden-Baden 2004, S. 13-71, hier S. 62-63.

3 Frank Schimmelfennig/Ulrich Sedelmeier: Theorizing EU Enlargement: Research Focus, Hypotheses, and the State of Research, in: Journal of European Public Policy 4/2002, S. 500-528, hier S. 503.

4 So bei Anna Michalski: The Enlarging European Union, in: Desmond Dinan (Hrsg.): Origins and Evolution of the European Union, Oxford 2006, S. 271-293, hier S. 272. 
die Stabilität der Gemeinschaft und ihr innerer Ausbau über alle diese Erweiterungsetappen hinweg erklärungsbedürftig. Diskussionswürdig sind schließlich die Langzeitfolgen der Erweiterungsrunden für den Gang der europäischen Integration.

\section{Zur Dynamik der Erweiterung}

Um die Dynamik der Erweiterung zu erklären, sollen drei Aspekte näher betrachtet werden, die sukzessive Ordnung des Felds möglicher Kandidaten, ihre Beitrittsmotive und die politische Identität der EG/EU.

\section{Die etappenweise Ordnung des Felds - Bildung und Aufhebung von Trennlinien}

Mit der Gründung der Gemeinschaft schufen die Sechs über die fundamentale blockpolitische Spaltung Europas hinaus, in die hinein ja bereits die Montanunion gegründet worden war, neue Trennlinien in Westeuropa: Die erste verlief zwischen Staaten, die supranationalgemeinschaftlich oder demgegenüber intergouvernemental-Souveränitäts-orientiert waren, die zweite zwischen jenen, die demokratische oder aber diktatorisch-autoritative Herrschaftssysteme etabliert hatten. Drittens grenzten sich nichtpaktgebundene und neutrale Länder gegenüber der EG ab, die ihnen als Teil der transatlantischen Blockstruktur galt. Entlang dieser drei Trennlinien formierte sich in Westeuropa das Feld der 16 Nicht-EG-Staaten und arrangierte sich im Laufe der Jahrzehnte in Bezug auf ,Brüssel' sukzessive neu. In vier Erweiterungsrunden ${ }^{5}$ sind die Trennlinien des westlichen Nachkriegseuropa, in zwei Schritten ist die Ost-West-Spaltung überwunden worden. ${ }^{6}$

Zuerst zur Ordnung des Feldes in Westeuropa: Gegenüber der Anziehungskraft der EG verloren Alternativgründungen sehr rasch an Attraktivität, wie die EFTA (,das Europa der 7“), die auf die Organisation des Freihandels auf dem Wege der Regierungszusammenarbeit ohne supranationale Vorkehrungen setzte. Obwohl das Vereinigte Königreich der maßgebliche Initiator der EFTA war, entwertete es die Neugründung sogleich durch seine stetig erklärten Ambitionen auf eine EWG-Mitgliedschaft. ${ }^{7}$ So entstand eine Art Dominoeffekt: Aufgrund der ambivalenten britischen Haltung geriet die gesamte EFTA in den Bannkreis der EWG. Schon 1961, zur Zeit des ersten britischen Antrags, war die Gemeinschaft sogleich mit einer Reihe förmlicher Bewerbungen und sondierender Anfragen konfrontiert, sodass sich Aussichten auf eine Verdopplung der Mitgliederzahl der Sechsergemeinschaft (,die 6 plus die 7“) bereits abzeichneten. ${ }^{8}$

Dem zweiten britischen Antrag von 1967 folgten zunächst Dänemark und Irland, die ihre Kandidaturen ausdrücklich vom Vereinigten Königreich abhängig machten. Nach der ersten Erweiterung 1973 blieben in Westeuropa jene 13 Staaten übrig, die sich aus politischen Grün-

5 Gemeint sind die Norderweiterung/erste EFTA-Erweiterung 1973 (Dänemark, Irland, Vereinigtes Königreich), die Süderweiterungen 1981 (Griechenland) und 1986 (Portugal, Spanien), die zweite EFTA-Erweiterung 1995 (Finnland, Österreich, Schweden) sowie die Osterweiterung 2004 (Estland, Lettland, Litauen, Malta, Tschechien, Polen, Slowakei, Slowenien, Ungarn, Zypern) und 2007 (Bulgarien, Rumänien).

6 Der Sonderfall der deutschen Einheit und der Ausdehnung der EG-Mitgliedschaft (ohne Beitrittsverhandlungen) auf das Beitrittsgebiet der neuen Bundesländer wird hier nicht weiter verfolgt. Vgl. Beate Kohler-Koch (Hrsg.): Die Osterweiterung der EG. Die Einbeziehung der ehemaligen DDR in die Gemeinschaft, Baden-Baden 1991; Barbara Lippert u.a.: Die EG und die neuen Bundesländer. Eine Erfolgsgeschichte von kurzer Dauer?, Bonn 1993.

7 So waren die EFTA-Märkte zu klein als Abnehmer für britische Industrieprodukte, aber hinzu kamen im Laufe der 1960er Jahre vermehrt politische Gründe, die für die EG-Mitgliedschaft sprachen.

8 Vgl. im Einzelnen zu den damaligen Interessenten Europäische Wirtschaftsgemeinschaft-Kommission: Bericht an das Europäische Parlament über den Stand der Verhandlungen mit dem Vereinigten Königreich, Brüssel, 26. Februar 1963, S. 98-100. 
den selbst ausschlossen, oder die von der EG politisch als nicht beitrittsfähig eingestuft wurden. Die Regierungen dieser Drittstaaten suchten jedoch durchweg aktiv privilegierte Bindungen und spezielle Beziehungen zur EWG. Das galt für Malta und Zypern, die in den frühen 1970er Jahren in ein engeres Verhältnis zur EG mittels der Assoziierung kamen und dann 1990 Beitrittsanträge stellten und für jene EFTA-Länder, mit denen ab 1973 Freihandels-Abkommen wirksam wurden und die Ende der 1980er Jahre die bislang höchste Ausformung der Assoziierung in Gestalt des EWR anstrebten. Die Deblockierung identitätspolitischer Vorbehalte von Neutralen und Nicht-paktgebundenen nach Ende des Ost-West-Konflikts erleichterte schließlich Schweden, Finnland und Österreich 1995 den Weg in die EU.

Unter den ursprünglich außen vor gebliebenen Drittstaaten Westeuropas sind als Sonderfälle zunächst Griechenland und die Türkei zu nennen. Die EWG-Staaten befürchteten, die Länder an der Südostflanke der NATO würden zwischen das Europa der Sechs und das EFTA-Europa fallen und suchten für Griechenland und die Türkei eine enge politisch-wirtschaftliche Anbindungsmöglichkeit. Mittels der sogenannten Beitrittsassoziierungen von 1961 und 1963 schuf die EWG eine Art Stufenplan zu späteren Mitgliedschaftsverhandlungen und war damit - vorrangig aus außenpolitischen Erwägungen - eine sehr langfristige politische Selbstbindung eingegangen. Sie verpflichtete sich mit Griechenland bis 1984 eine Zollunion zu schaffen, während sie mit der Türkei zunächst nur eine diesbezügliche Vorbereitungszeit ins Auge fasste. ${ }^{9}$

Besonders gelagerte Fälle waren auch Spanien und Portugal, die sich wegen ihrer autoritären politischen Systeme bis zur Mitte der 1970er Jahre selbst von einer EG-Mitgliedschaft ausgeschlossen hatten (was über weite Strecken auch auf die Türkei - Militärputsch 1960, 1971, 1980 - und zwischen 1967 und 1974 auch auf Griechenland zutraf), obwohl sie sicherheitspolitisch (Portugal und Türkei waren seit 1949 beziehungsweise 1952 NATO-Mitglieder) und/oder wirtschaftlich zumindest teilweise in den Westen integriert waren. ${ }^{10}$ Beim Beitritt Griechenlands, Spaniens und Portugals (1981/86) ging es erstmals für die EG darum, das ,ganze freie Westeuropa' in ihren Wirkungskreis bis hin zur Mitgliedschaft einzubeziehen. Die damals innerhalb der EG umstrittene zügige Aufnahme Griechenlands präjudizierte eine Aufnahme der beiden jungen iberischen Demokratien. ${ }^{11}$ Mit dieser in zwei Etappen vollzogenen Süderweiterung wurde die Trennlinie zwischen dem demokratischen Westeuropa und dem autoritär-diktatorischen Süden überwunden.

Die „Leftovers des alten Westens“ sind heute: die Türkei, die Schweiz, Liechtenstein, Norwegen und Island. ${ }^{12}$

Im Gegensatz zur etappenweisen Aufnahme der westeuropäischen Staaten gelangte das freie Mittel- und Osteuropa 2004/07 quasi auf einen Schlag durch eine Big Bang-Erweiterung in die EU. Mit dem Fall des Eisernen Vorhangs 1989 wurde die ,Jalta“-Trennlinie zwischen Ost und West mit einem Mal aufgehoben und der „Ostblock“ verschwand von der politischen Landkarte. Die Erweiterung nach Osten folgte - vergleichbar nur mit der Gründung der Montanunion - auf einen externen Schock und eine weltpolitische Zäsur im Gang der europäischen Integration. ${ }^{13}$ Die wiedererlangte Souveränität und der Staatszerfall der drei nach dem Ersten Weltkrieg gegründeten Föderationen - UdSSR, Jugoslawien und Tsche-

9 Vgl. ausführlich Sena Ceylanoglu: Europäische Wirtschaftsgemeinschaft, Griechenland und die Türkei. Die Assoziationsabkommen im Vergleich (1959-1963), Baden-Baden 2004.

10 So waren Spanien und Portugal ab 1961 Mitglieder der OECD, zudem nahm Portugal am Marshallplan teil und war seit 1960 Mitglied der EFTA.

11 Anfangs war eine Aufnahme der drei Staaten zum selben Zeitpunkt angestrebt worden.

12 Sowie die sogenannten Mikrostaaten Andorra, Monaco, San Marino und der Heilige Stuhl.

13 Siehe dazu den Beitrag von Link in diesem Heft. 
choslowakei - ließ die Zahl der neuen potenziellen Bewerber um eine EU-Mitgliedschaft Anfang der 1990er Jahre auf rund 20 Staaten hochschnellen. Sobald der Osten entriegelt war, zerfiel die Gegenorganisation, der RGW, und eine „EG II“ des Ostens als Alternativoption wurde von keiner politischen Kraft angestrebt. Auch in der Folgezeit entwickelte sich nur eine schwache interregionale Kooperation in Ostmittel- und Südosteuropa. ${ }^{14}$ Für eine Regionalbildung neben der EU gab es kein attraktives und legitimiertes politisches Kraftfeld. Dem Ansturm auf die EG/EU kam nur der auf die NATO gleich. ${ }^{15}$

Die „Leftovers des neuen Ostens“ sind heute die seitens der EU mit einer Beitrittsperspektive ausgestatteten Staaten Kroatien, Mazedonien, Serbien, Bosnien-Herzegowina, Montenegro und Albanien, die überwiegend wegen der jugoslawischen Nachfolgekriege in den 1990ern vom EU-Beitritt ausgeschlossen waren. Obwohl die EU ihrerseits der Ukraine, Belarus, Moldova und den drei Ländern des Südkaukasus (noch) keine Beitrittsperspektive zugesprochen hat, zählen sie, sowie im Prinzip auch Russland, zum Kreis potenzieller Mitglieder.

Nach 1989 drängte sich für die EU die neue Frage der geografischen Grenzziehung auf eine Frage, die bis dato entweder wegen natürlicher Grenzen im Westen, Norden und Süden irrelevant schien, oder durch die Systemgrenze im Osten entschieden war. ${ }^{16}$ Tendenziell wurde - inspiriert durch die Süderweiterung - die EG bereits in den 1970ern und 1980ern als „Kernzelle der Freiheit in Europa“17 verstanden und das Szenarium einer auf den gesamten Kontinent ausgreifenden Erweiterung der EG diskutiert. So prophezeite Kommissar Haferkamp 1977, man werde ,....in Europa von einem säkularen Einigungsprozeß ausgehen müssen, der schließlich den ganzen Kontinent einbeziehen wird..." 18 Heute bedeutet dies, eine EU mit bis zu 44 Mitgliedstaaten in den Blick zu nehmen. ${ }^{19}$

\section{Beitrittsmotive}

Die aus den Römischen Verträgen entwickelte EWG/EG übte also beständig eine große Anziehungskraft auf die außen vor gebliebenen Staaten aus. Die schiere Existenz einer Gemeinschaft, die eine strukturgebende Bedeutung für die Ordnung Westeuropas hatte, zwang die Nicht-EWG-Länder im Westen Europas aus politischen und/oder wirtschaftlichen Gründen verbindliche und förmliche Regelungen für ihre Beziehungen zu dem neuen Akteur zu suchen. Am weitestgehenden geschah dies durch den Antrag auf Mitgliedschaft. Die Beitrittsmotive der Kandidaten variierten zwar, sie lassen sich aber - stark vereinfacht - drei Sachbereichen der Politik - Wohlfahrt, Sicherheit und Herrschaft - zuordnen. ${ }^{20}$

14 Vgl. Egbert Jahn: Ausdehnung und Überdehnung. Von der Integrationskonkurrenz zum Ende der europäischen Integrationsfähigkeit, in: Osteuropa 2-3/2007, S. 35-55.

15 Vgl. Stuart Croft u.a.: The Enlargement of Europe, Manchester/New York 1999.

16 Aus geografischen Gründen wies die EG den Antrag von Marokko 1987 zurück. Vgl. zur Ablehnung einer abschließenden Festlegung geografischer Grenzen durchgehend die Argumentation der Kommission: Europäische Kommission: Europa und die Problematik der Erweiterung. Bericht der Kommission an den Europäischen Rat von Lissabon, 26.-27. Juni 1992, in: Europa-Archiv, 15-16/1992, S. D508-D514, hier S. D509 (Punkt 7); sowie Europäische Kommission: Erweiterungsstrategie und wichtigste Herausforderungen für den Zeitraum 2006 2007 mit Sonderbericht über die Fähigkeit der EU zur Integration neuer Mitglieder, KOM(2006) 249, S. 18-27.

17 Außenminister Genscher am 19.1.1977 vor dem 8. Deutschen Bundestag, in: Deutscher Bundestag: Verhandlungen des Deutschen Bundestages, Stenographische Berichte, Band 100, Bonn 1977, S. 149.

18 Wilhelm Haferkamp: Chancen und Risiken der zweiten EG-Erweiterung, in: Europa-Archiv, 19/1977, S. 617626, hier S. 617.

19 Vgl. Graham Avery: An ever-wider Europe? Where will the EU's borders end?, in: Challenge Europe 16, Februar 2007, S. 101-109.

20 Vgl. generell zu den jeweiligen Motivlagen Christopher Preston: Enlargement and Integration in the European Union, London 1997; überblicksweise Timm Beichelt: Die Europäische Union nach der Osterweiterung, Wiesbaden 2004, S. 20-34 sowie länderspezifisch Jürgen Elvert/Wolfram Kaiser (Hrsg.): European Union Enlargement. A Comparative History, London/New York 2004. 
An erster Stelle ist der Sachbereich Wohlfahrt anzuführen, denn alle Kandidaten sämtlicher Erweiterungsrunden teilten das zentrale Interesse an der Wohlstandsmaximierung. Überragend war der Wunsch, dem Erfolg der Europäischen (Wirtschafts-)Gemeinschaft mit dem Gemeinsamen Markt, später dem Binnenmarkt, als Herzstück beizutreten. Die Anziehungskraft des Marktes ging bei den Bewerbern mit der Sorge einher, von diesem Wirtschafts- und Währungs-Block ausgeschlossen zu werden. Wichtig war auch die Zentralität der Bundesrepublik Deutschland, mit der die Nachbarn in Westeuropa enge Handelsverflechtungen unterhielten, was ihre Orientierung auf den EG-Markt noch verstärkte. Das galt in der ersten Beitrittsrunde für Dänemark ebenso wie später beispielsweise für Österreich und viele Länder Mittel- und Osteuropas. Es war mithin die Zugehörigkeit zu einer wirtschaftlich und politisch dynamischen Gemeinschaft, die auch die relativ reichen EFTA-Länder in aller erster Linie anzog. Zudem spielte bei der Süderweiterung von 1981 und 1986 ebenso wie bei der Osterweiterung 2004/2007 die Aussicht auf Finanztransfers eine Rolle. Bei allen Erweiterungsrunden, besonders aber bei der Osterweiterung nutzten die Regierungen und wirtschaftlichen Akteure der Kandidaten den Beitritt als Modernisierungshebel. So begründete schon die britische Regierung ihren Beitrittsantrag mit dem Ziel des Strukturwandels und der zusätzlichen Wachstumsimpulse. ${ }^{21}$

Die mit der Aussicht auf Wohlstandsmaximierung verbundenen Interessen und Präferenzen der Bewerber betonen vor allem Vertreter des liberalen Intergouvernementalismus, um die Attraktivität der EU zu erklären. ${ }^{22}$ Demgegenüber rekurrieren Vertreter der (neo-)realistischen Schule in erster Linie auf Sicherheitsinteressen, auch wenn die EG keine militärische Sicherheit bot. In allen Erweiterungsrunden spielte aber auf Seiten der Kandidaten die Sorge vor einer Peripherisierung, Marginalisierung oder gar Isolation eine wichtige Rolle. Es ging darum, die Stellung und den Einfluss des eigenen Landes im internationalen System zu sichern. So suchte die britische Regierung 1967 auch deshalb um den Beitritt nach, weil sie auf politischem und wirtschaftlichem Feld Vorkehrungen gegen einen weiteren macht- und weltpolitischen Abstieg Britanniens treffen wollte. Griechenland ging es um eine Positionsverbesserung im regionalen Sicherheitsraum, vor allem um einen Vorsprung vis-à-vis der Türkei. Gerade die Nord- und die zweite EFTA-Erweiterung brachten die Sorgen der Nachzügler zum Ausdruck, außerhalb der EG stetig an Einfluss zu verlieren und zugleich einen immer höheren politischen Preis für den Beitritt zahlen zu müssen. Auch wenn sämtliche zehn Länder der Osterweiterung 2004/07 zuvor oder gleichzeitig der NATO beigetreten waren, so galt ihnen doch die EU-Mitgliedschaft als eine zusätzliche Sicherheitsgarantie und politische Arena, die ihren Einfluss in den internationalen Beziehungen beträchtlich vergröBern wird. ${ }^{23}$ Die EG/EU-Mitgliedschaft wurde so zum Vehikel, mit der sich zunächst die Staaten Westeuropas und dann auch Mittel- und Osteuropas im internationalen System (kollektiv) zu behaupten suchten.

Uneinheitlicher fällt die Motivlage in Bezug auf den Sachbereich Herrschaft aus. Mit Fragen der politischen Identität befassen sich besonders Analysen zur Erweiterung aus Sicht des (Sozial-)Konstruktivismus. Die Osterweiterung stand explizit unter dem Motto der

21 Vgl. Statement by the Prime Minister, Harold Wilson, before the House of Commons, 2 May 1967, abrufbar unter: http://aei.pitt.edu/5779 (letzter Zugriff: 05.09.2007).

22 Zur Analyse der Erweiterung aus Sicht unterschiedlicher Integrationstheorien vgl. den Überblick bei Lippert: Glanzloser Arbeitserfolg, 2004, S. 15-21 sowie Frank Schimmelfennig/Ulrich Sedelmeier: The Study of European Union Enlargement: Theoretical Approaches and Empirical Findings, in: Michelle Cini/Angela K. Bourne (Hrsg.): European Union Studies, Houndmills, Basingstoke und Hampshire 2006, S. 96-116.

23 Dazu Mathias Jopp/Barbara Lippert/Elfriede Regelsberger: Europäische Außen- und Sicherheitspolitik nach der Erweiterung - politische und institutionelle Herausforderungen und Lösungsansätze, in: Lippert (Hrsg.): Bilanz und Folgeprobleme, 2004, S. 241-260. 
„Rückkehr nach Europa“. Aus Sicht der Kandidaten der Süd- und Osterweiterung (und der EU) galt der Beitritt als Beitrag zur Konsolidierung junger Demokratien. Ein gewünschter Effekt, der besonders bei einer relativ langen und intensiven Heranführungszeit zum Tragen kommen konnte, war zumindest bis zum Beitritt die Stärkung einer demokratischen politischen Mitte (Sozialisierungsfunktion) in den jungen Demokratien. ${ }^{24}$ Anders sah die Motivlage bei den bereits reichen und/oder konsolidierten neuen Mitgliedstaaten aus, etwa in Britannien, Dänemark und Schweden. Dort war die politische Identität der Union eher als ein ,,cultural“ und ,political mismatch“ mit der gefestigten nationalen Identität und der politischen Kultur (wie etwa die Vorstellung von der „British singularity“) wahrgenommen worden. ${ }^{25}$

\section{Selbstverständnis und Vertragsgrundlagen der EG}

Neben dem Beitrittsbegehren von interessierten Drittstaaten haben auch endogene Faktoren zur Dynamik der Erweiterung beigetragen. Die deklarierte und in den Verträgen kodifizierte Offenheit der Gemeinschaften verstärkte in Vergangenheit und Gegenwart die Dynamik zur Erweiterung. Das Thema der Aufnahme weiterer Mitglieder war vor, während und nach der Gründung der drei europäischen Gemeinschaften immerfort präsent. Die Unabgeschlossenheit und das Prozesshafte, die bereits die Vertiefung der Integrationsgemeinschaften kennzeichnen, gelten auch für deren Erweiterung.

Ein wichtiges Signal zur Erweiterung kam damit von der EG selbst: Die Sechsergemeinschaft begann auch in Bezug auf ihre Mitglieder als unvollendete Gemeinschaft, denn sie wurde von den sechs Gründerstaaten selbst keineswegs als Endstufe angesehen. Die Gretchenfrage war von Anbeginn: Wie steht es mit dem Vereinigten Königreich? Besonders Deutschland, die Niederlande und Belgien hätten das Vereinigte Königreich allein schon aus wirtschaftlichen Gründen gerne frühzeitig in der EWG dabei gehabt. Britannien war das Land, um das die EG selbst am nachdrücklichsten als neues Mitglied geworben hat. ${ }^{26}$ Elvert sieht sogar erst mit der Norderweiterung 1973 die Gründungsphase der EG zu ihrem Abschluss gelangt. ${ }^{27}$

Bewerber benötigen unter den Mitgliedstaaten ,Champions', die ihr Anliegen unterstützen. Seit der ersten Erweiterung gibt es mit Deutschland und dem Vereinigten Königreich zwei große Mitgliedstaaten, die zu den nachdrücklichsten Befürwortern aller Erweiterungsrunden bis 2007 zählten. ${ }^{28}$ Eine Schwierigkeit für die deutsche Europapolitik blieb allerdings, dass es keine gemeinsame deutsch-britische Vertiefungsagenda gab, die zu einer Doppelstrategie von Vertiefung und Erweiterung heranreifen konnte. ${ }^{29}$ Für die Eröffnung und den Abschluss von Verhandlungen blieb ohnehin eine deutsch-französische Übereinkunft ausschlaggebend ${ }^{30}$ - siehe auch jetzt den aktuellen Fall der Türkei. Bei allen Erweite-

24 Vgl. Milada Anna Vachudova: Europe Undivided. Democracy, Leverage, and Integration after Communism, Oxford 2005.

25 Vgl. die Länderanalysen in Kaiser/Elvert: European Union Enlargement. A Comparative History, 2004.

26 So war von einer „Beitrittskrise“ die Rede angesichts der französischen Blockade von Verhandlungen bis 1969, vgl. Jürgen Mittag/Wolfgang Wessels: Die Gipfelkonferenzen von Den Haag (1969) und Paris (1972): Meilensteine für Entwicklungstrends der Europäischen Union?, in: Franz Knipping/Mathias Schönwald (Hrsg.): Aufbruch zum Europa der zweiten Generation - Die europäische Einigung 1969-1984, Trier 2004, S. 3-27, hier S. 15; warnend vor einer schweren Krise auch Kanzler Kiesinger am 11.3.1968 vor dem 5. Deutschen Bundestag, in: Deutscher Bundestag: Verhandlungen des Deutschen Bundestages, Stenographische Berichte, Band 66, Bonn 1968, S. 8169-8190.

27 Vgl. Jürgen Elvert: Die europäische Integration, Darmstadt 2006, S. 35-88.

28 Das galt bei der Regierung Thatcher allerdings nicht für die Aufnahme der neuen Bundesländer.

29 Vgl. Barbara Lippert u.a.: British and German Interests in EU Enlargement, London/New York 2001. 
rungen (außer Griechenland) trat Frankreich zunächst als Gegner oder zumindest als Bremser in Erscheinung.

Das gemeinschaftliche Primärrecht zur Erweiterung blieb statisch und unterhalb der Mitgliedschaft blieb es vornehmlich beim variantenreichen Status der Assoziierung. Das hatte Gründe: So hatte die EG 1968 angesichts des französischen Vetos gegen eine Aufnahme von Beitrittsverhandlungen mit dem Vereinigten Königreich erste Vorbereitungen für ein Spezialarrangement auf den Feldern Handel (Präferenzabkommen) und technologische Zusammenarbeit in Angriff genommen. ${ }^{31}$ Die Vorarbeiten für eine solche Interimslösung zur Umgehung des französischen Vetos wurden aber schon Mitte 1969 wieder eingestellt, nachdem der Rat die Kommission aufgefordert hatte, ihre Stellungnahmen zum britischen Beitrittsgesuch von 1967 im Vorlauf zum Haager Gipfel zu überarbeiten. Die Suche nach einer Akkordierung mit dem EG-Recht durch Spezialarrangements galt als politisch heikel für Drittstaaten (da diese keine Entscheidungsteilhabe hatten) und als rechtlich kompliziert für die EWG und Mitgliedstaaten: ,...for countries which are sufficiently developed economically and possess institutions and regimes comparable with those of the founder States, the Community has always considered that full membership was the arrangement that accorded best with the objectives of the Treaties. The drawbacks of association agreements or preferential agreements with such countries are well known; first, these countries might in certain cases have to comply with decisions in the taking of which they had had no part, and second, the commitments on consultation and the multiplicity of special arrangements would make for inextricable complications for the Community." 32 Ähnlich stellte sich später vor allem am Beispiel des EWR und angesichts eines beträchtlich angewachsenen Acquis communautaire sowie komplexer Entscheidungsverfahren heraus, dass die Schaffung einer Zwischenstufe beide Seiten mit größeren Problemen der Gesamtkonstruktion und der Selektion des Acquis konfrontierte, als wenn man direkt Mitgliedschaftsverhandlungen führte. Daraus zogen seit Ende der 1960er Jahre beide Seiten immer wieder den Schluss, dass die Aufnahme neuer Mitglieder beziehungsweise der Beitritt als Catch-all-Lösung einfacher zu bewerkstelligen sei als Zwischenlösungen.

Sofern Vertragsrevisionen und Schritte zur weiteren politischen Integration den intergouvernementalen Ausbau der EG/EU - etwa durch die Einführung der EPZ/GASP oder des Europäischen Rates als neuer Institution - vorantrieben, wurde die Mitgliedschaft auch für jene Länder, die grundsätzlich souveränitätsschonende Lösungen präferierten, zusätzlich attraktiv. Auch dies waren Impulse für die Erweiterung, die direkt der inneren Entwicklung der EG/EU entsprangen.

Ausgehend von den kaum modifizierten Bestimmungen über die Aufnahme neuer Mitglieder machten die Mitgliedstaaten im Zeitraum von 1973 bis 2007 in erster Linie politische Motive für die Erweiterung geltend, wobei einerseits die Gründungsmotive der Gemeinschaften einen wichtigen Bezugspunkt und Legitimationsquelle bildeten und andererseits der allgemeine integrationspolitische und internationale Kontext eine zentrale

30 Vgl. für die erste und zweite Erweiterung z.B. Haig Simonian: The Privileged Partnership. Franco-German Relations in the European Community 1969-1984, Oxford 1985, S. 2 und 78/79.

31 Vgl. zu den Memoranda und Vorschlägen der italienischen Regierung und der Bundesregierung Commission of the European Communities: The Enlarged Community. Outcome of the negotiations with the applicant States, Brussels, 22 January 1972, S. 16 sowie Herbert Müller-Roschach: Die deutsche Europapolitik 19491977. Eine politische Chronik, Bonn 1980, S. 200.

32 Commission of the European Communities: Opinion concerning the Applications for Membership from the United Kingdom, Ireland, Denmark and Norway submitted under Articles 237 of the EEC Treaty, 205 of the Euratom Treaty, and 98 of the EESC Treaty, 1 October 1969, in: Bulletin of the European Communities, Supplement No. 9/10, Luxemburg 1969, S. 26. Vgl. auch Müller-Roschach: Europapolitik, 1980, S. 194-196. 
Rolle spielte. Insofern waren identitätspolitische Gesichtspunkte wirksam. Die Aufnahmebereitschaft war innerhalb der EG/EU weniger als eine Frage des Prinzips (wie noch von de Gaulle angesichts des britischen Antrags) behandelt worden, sondern in der Regel als eine Frage des Wie und Wann: Im Vordergrund stand der Abwägungsprozess im Hinblick auf die mutmaßliche Verträglichkeit und die Wünschbarkeit der potenziellen politischen und wirtschaftlichen Folgen für die erweiterte Gemeinschaft. ${ }^{33}$ Seitdem schlagen sich diese länder-, politikfeld- und branchenspezifischen Kosten-Nutzen-Abschätzungen in Wirkungsstudien und in einem kleinteiligen Bargaining unter den Mitgliedstaaten im Hinblick auf die Festlegung Gemeinsamer Positionen für die Verhandlungen mit den Bewerbern nieder. ${ }^{34}$ Die zumeist ungleich unter den Mitgliedstaaten verteilten Kosten der Erweiterung werden durch Sidepayments und Perspektivangebote für die innere Entwicklung der EU kompensiert. So ergeben sich weitere Impulse für Reformen und den Ausbau bestehender Regelungen im Rahmen von Koppelgeschäften (Paketlösungen). ${ }^{35}$

Die Erweiterung als ,Feind" der Vertiefung wird in integrationsfreundlichen Mitgliedstaaten und politischen Kreisen mindestens ebenso heftig diskutiert wie sie andererseits als Impulsgeber für eine schnellere und umfassendere Reform und Fortentwicklung der Integration befürwortet wird. Die Parole „Strengthening and enlargement“" gab die Kommission schon 1969 für die erste Erweiterung aus. ${ }^{36}$ Deshalb forderte sie, dass sich die Kandidaten über den aktuellen Acquis hinaus zur weiteren Vertiefung bekennen müssten. Denn die Kommission und auch das Europäische Parlament sahen sehr wohl Gefahren für den $\mathrm{Zu}$ sammenhalt der Union, denen mit einer ,better institutional balance“"37 entgegengesteuert werden sollte. Skeptiker erwarteten schon mit Blick auf die Süderweiterung einen Ausbau der intergouvernementalen, konföderalen Ansätze und den Wandel von der „Schicksalsgemeinschaft" zum Problemlösungsverband und einer Zweckgemeinschaft. Die Tendenz zu einer konföderal verstandenen Staatenverbindung $(, \ldots$ die bereits von Frankreich verfolgt wird und seit der ersten Erweiterung zunehmend Unterstützung findet ${ }^{\text {“38) }}$ ) würde durch die Süderweiterung verstärkt. ,[A]nstelle der ursprünglich angestrebten politischen Einheit als Schicksalsgemeinschaft [tritt] eine in wesentlichen Bereichen als Staatenzusammenarbeit organisierte Vereinigung zur gemeinsamen Bewältigung bestimmter politischer und wirtschaftlicher Aufgaben... "39 Als die schließlich zum Abschluss der Einheitlichen Europäischen Akte führende Regierungskonferenz einberufen werden sollte, stimmten denn auch 1985 drei Nicht-Gründerstaaten - Griechenland, das Vereinigte Königreich und Dänemark diesem Beschluss des Europäischen Rates nicht zu.

Die EG/EU verstand es im Verlauf, aber auch in der, Verarbeitung' der Erweiterungsrunden jeweils eine ,Erzählung' zu entwickeln, die eine ausreichende Unterstützung in der EU für die Aufnahme neuer Mitglieder trotz erkennbarer Anpassungserfordernisse und anderer Kosten ermöglichen sollte. Dabei waren die Narrative unterschiedlich eingängig und reso-

33 Vgl. umfassend William Wallace/Geoffrey Edwards/Loukas Tsoukalis: Eine Gemeinschaft der Zwölf: Die Europäische Gemeinschaft und ihre Erweiterung nach Süden, in: Europa-Archiv 19/1977, S. 627-690.

34 Vgl. vor allem die 1997 von der Kommission vorgelegte Agenda 2007. Dazu mit Nachweisen Lippert: Glanzloser Arbeitserfolg, 2004, S. 35-47.

35 Vgl. Michalski: Enlarging, 2006, S. 285; Barbara Lippert: Die Erweiterungspolitik der Europäischen Union Stabilitätsexport mit Risiken, in: Barbara Lippert (Hrsg.): Osterweiterung der Europäischen Union - die doppelte Reifeprüfung, Bonn 2000, S. 105-164, hier S. 153.

36 Commission: Opinion, 1969, S. 29-30.

37 Ebenda, S. 32

38 Ulrich Everling: Integrationspolitische Probleme der Erweiterung der EG, in: Hajo Hasenpflug/Beate Kohler (Hrsg.): Die Süd-Erweiterung der Europäischen Gemeinschaft. Wende oder Ende der Integration?, Hamburg 1977, S. 61-87, hier S. 66.

39 Ebenda, S. 68. 
nanzkräftig: Das Narrativ der Norderweiterung war die ,Politische Selbstbehauptung und Emanzipation Westeuropas', die Erzählung zur Süderweiterung galt der EG als ,Demokratie- und Entwicklungsgemeinschaft (Kohäsion)', das Narrativ der Osterweiterung war die „Wiedervereinigung des Kontinents“. Dagegen blieb das Narrativ der EU zur zweiten EFTA-Erweiterung blass und resonanzarm. Es kreiste am ehesten um die ,Arrondierung des alten Westens und die Stärkung durch nettozahlende Wohlfahrtsstaaten'. Bei den künftig anstehenden Erweiterungen um Staaten des westlichen Balkans bietet sich das Narrativ der EU als ,Pazifizierungsmacht` an, und im Falle der Türkei hebt bereits die Erzählung von der ,Brücke zwischen dem aufgeklärten Westen und dem Islam‘ an. Zum jeweiligen Zeitpunkt trugen diese Narrative zur Dynamik und Akzeptanz des EU-Erweiterungsprozesses bei. Für Osteuropa und den Südkaukasus ist bislang noch kein starker Erzählfaden „,gesponnen“ worden. Im Hinblick auf die Legitimation künftiger Erweiterungsschritte werden Fähigkeiten und Strategien des Argumentierens und Überzeugens immer wichtiger, sowohl im Diskurs der politischen Eliten untereinander als auch im Hinblick auf eine skeptischer werdende Bevölkerung in vielen Mitgliedstaaten.

\section{Stabilität plus Ausbau der Gemeinschaft}

\section{Stärke und Prägekraft der Sechsergemeinschaft}

Die primäre Voraussetzung für jegliche über den Kreis der Sechs hinausgehende Erweiterung war die durch die Kernmitglieder Frankreich und Deutschland gesicherte politische Tragfähigkeit der Gemeinschaft. Die beiden Schlüsselstaaten für das integrative Gleichgewicht in Westeuropa bildeten den politischen Kern einer erweiterungsfähigen Gemeinschaft.

Eine weitere wichtige Voraussetzung für die erste Erweiterung war, dass die EWG ihr in den Römischen Verträgen vereinbartes Programm zur Schaffung des Gemeinsamen Marktes vor der Aufnahme neuer Mitglieder 1973 weitgehend zu Ende führen konnte, ${ }^{40}$ dass der Vertrag über die Fusion der Organe 1967 in Kraft getreten war (damit kam nur ein gleichzeitiger Beitritt zu allen drei Gemeinschaften in Frage) und die Gemeinschaft Anlauf zu einer neuen Etappe nahm, für die der Gipfel von Den Haag 1969 den Dreiklang „Vollendung, Vertiefung, Erweiterung “ ausgerufen hatte. Die Gemeinschaft hatte auch bereits ihre größte ,Verfassungskrise“ in der Zeit der Politik des leeren Stuhls hinter sich. Sie war somit schon in der Phase der Desillusionierung angelangt, aus der jedoch auch die Hoffnung erwuchs, die Aufnahme neuer Mitglieder als Schwungrad für den inneren Ausbau und die Vertiefung nutzen und so die damalige Stagnation überwinden zu können. Das heißt, der Zeitpunkt der ersten Erweiterung war angesichts der fortgeschrittenen Konsolidierung des von den Sechs geprägten Systems der Römischen Verträge gut gewählt.

\section{Die „Erweiterungsdoktrin“}

Die mit der Norderweiterung etablierten Prinzipien der Erweiterung und die administrativen Vorkehrungen für die Beitrittsverhandlungen erwiesen sich zudem als Stabilitätsfakto-

40 Insbesondere die sog. Übergangsperiode zur Zollunion, zur gemeinsamen Außenhandelspolitik, zum System der Gemeinsamen Agrarpolitik. So war beim Haager Gipfel der „Eintritt in die Endphase des gemeinsamen Markts" festgehalten worden. Die Situation unterschied sich deutlich von der beim ersten britischen Antrag. Die Kommission bemerkte dazu in ihrem Bericht an das Europäische Parlament nach Abbruch der Verhandlungen 1963: „Aber auch die Tatsache, daß in anderen Bereichen der Vertrag erst zum Teil durchgeführt war, und daß sich ganz allgemein seine Verwirklichung in den verschiedenen Bereichen noch in einem Zwischenstadium befand, dürfte die Verhandlungen nicht unwesentlich erschwert haben." Europäische Wirtschaftsgemeinschaft - Kommission: Bericht, S. 113-114. 
ren für die alte und die künftig erweiterte Gemeinschaft. Die erste Erweiterung legte die Grundlagen für das klassische Erweiterungsverfahren der EU, ${ }^{41}$ das vor allem durch die wiederholte Praxis, weniger durch formale Festlegungen zu einer Art „Erweiterungsdoktrin“ heranreifte.

Grundlegendes Prinzip war die Kompatibilität der politischen Ordnungen zwischen der EG und dem Bewerberland. So kann der vom Europäischen Parlament mit großer Mehrheit angenommene Birkelbach-Bericht von 1962 als Vorläufer der Kopenhagener Kriterien von 1993 gelten. Im Bericht wird festgestellt: „Als Voraussetzung für einen Beitritt muss das Bestehen einer demokratischen Staatsform im Sinne einer freiheitlichen politischen Grundordnung gewährleistet sein. Staaten, deren Regierungen keine demokratische Legitimation besitzen und deren Völker weder direkt noch durch frei gewählte Repräsentanten an der politischen Willensbildung mitwirken, können nicht beanspruchen, in den Kreis der Völker aufgenommen zu werden, die sich in den Europäischen Gemeinschaften zusammengeschlossen haben." ${ }^{" 42}$ Kommission und Rat teilten dieses Prinzip, und die Kommission bezog wie das Europäische Parlament diese Standards gleichermaßen auf die Prüfung von möglichen Assoziierungen nach Artikel 238 EWG- und 206 Euratom-Vertrag. ${ }^{43}$ Im Laufe der Jahrzehnte, ${ }^{44}$ besonders aber in den 1990er Jahren, wurden die politischen Kriterien zunächst im politischen Diskurs immer prägnanter formuliert und fanden schließlich auch in einem in dieser Hinsicht erweiterten Primärrecht (Art. 6 EUV Amsterdam) ${ }^{45}$ sowie in den 1993 vom Europäischen Rat in Kopenhagen deklarierten Beitrittskriterien ihren Niederschlag. Die politischen Kriterien gewannen aber in den 1990er Jahren vor allem durch die Verdichtung der politischen Identitätsprozesse auf dem Weg zur Europäischen Union in der Nachfolge des Vertrags von Maastricht nachhaltig Bedeutung. ${ }^{46}$ Die Sorge um die Funktions- und Integrationsfähigkeit der EG/EU, aber auch die Option einer restriktiven Auslegung der Erweiterungsmöglichkeit waren ein wichtiges Motiv für derartige Präzisierungen der Aufnahmebedingungen. Die im Reformvertrag vorgesehene Überarbeitung des Artikels 49 EUV setzt hier an und zielt auf eine transparentere und ausdrückliche Darlegung von Beitrittskriterien, die eine vorherige Reflexion und Abstimmung unter den Mitgliedstaaten über die konkreten Aufnahmebedingungen erzwingen und angeblichen Automatismen entgegenwirken soll. Der

41 Vgl. zur Norderweiterung ausführlich Klaus Otto Nass: Englands Aufbruch nach Europa. Ein erster Überblick über die Beitrittsverhandlungen, Bonn 1971, besonders S. 41-63. Beschluß des Rats vom 9.6.1970 über das bei den Beitrittsverhandlungen anzuwendende Verfahren, abgedruckt in: Europa-Archiv 15-16/1070, D350. Vgl. zum sich entwickelnden klassischen Erweiterungsverfahren Christopher Preston: Obstacles to EU-Enlargement: The Classical Community Method and the Prospects for a Wider Europe, in: Journal of Common Market Studies 3/1995, S. 451-463; Lippert: Glanzloser Arbeitserfolg, 2004, S. 31-35.

42 Willi Birkelbach: Bericht im Namen des Politischen Ausschusses über die politischen und institutionellen Aspekte des Beitritts zur Gemeinschaft oder der Assoziierung mit ihr, in: Europäisches Parlament, Sitzungsdokumente 1961/62, 15.1.1962, Dokument 122, S. 5.

43 Vgl. Commission of the European Communities: Opinion on the Applications for Membership received form the United Kingdom, Ireland, Denmark and Norway, 1967, S. 16.

44 So etwa Außenminister Genscher am 19.1.1977 vor dem 8. Deutschen Bundestag, in: Deutscher Bundestag: Verhandlungen des Deutschen Bundestages, Stenographische Berichte, Band 100, Bonn 1977, S. 148: „Eine Reihe von Ländern ... erfüllen heute durch eine Veränderung ihrer politischen Verhältnisse die politischen Voraussetzungen für die Mitgliedschaft in der Europäischen Gemeinschaft. Ich nenne Griechenland, ich nenne Portugal, ich nenne Spanien. Wir alle wissen, daß diese Länder durch die Präambel, durch den Sinn der Römischen Verträge eine Beitrittsperspektive eröffnet bekommen haben. Ja, sie haben, so weit sie assoziiert sind, sogar einen Anspruch auf Beitritt. Deshalb war es richtig, daß sich die Bundesregierung zu den Befürwortern der Aufnahme von Beitrittsverhandlungen mit Griechenland gemacht hat... ." (Hervorh. B.L.).

45 „Jeder europäische Staat, der die in Artikel 6 Absatz 1 genannten Grundsätze [Freiheit, Demokratie, Achtung der Menschenrechte und Grundfreiheiten, Rechtsstaatlichkeit; Zusatz B.L.] achtet, kann beantragen, Mitglied der Union zu werden." Art. 49 EUV (in der Fassung vom 10.11.1997).

46 Schimmelfennig/Sedelmeier: European Union Enlargement, 2006. 
neu einzufügende Satz „Die vom Europäischen Rat vereinbarten Kriterien werden berücksichtigt ${ }^{* 47}$ kann über die Kopenhagener Kriterien hinaus auf weitere Einlassungen des Europäischen Rates aus der Vergangenheit und der Zukunft bezogen werden. Damit hätte die EU eine größere Flexibilität, Beitrittskriterien den Umständen entsprechend zu formulieren, sie müsste aber zunächst in der Lage sein, sich auf solche ausdrücklich zu einigen.

Das zweite Prinzip der klassischen Erweiterungsdoktrin ist, dass der Acquis in Gänze einschließlich der politischen Perspektivbeschlüsse, also der politischen Zielsetzungen der Verträge von jedem Kandidaten zu übernehmen ist. In diesem Sinne argumentierte die Kommission schon in ihrer Stellungnahme von 1969 und so bekräftigte es der Gipfel von Den Haag. ${ }^{48}$ Der Ratsvorsitzende Harmel unterstrich am 30. Juni 1970 bei Eröffnung der Beitrittsverhandlungen mit dem Vereinigten Königreich, dass es keine Änderungen von bestehenden Vorschriften, sondern nur befristete Übergangsregelungen als Lösung von Problemen (auf beiden Seiten) geben könne. ${ }^{49}$ Es sollte generell gelten, dass Kandidaten keine Bedingungen an die EG stellen können, wie dies noch 1961 von der Regierung Macmillan im Hinblick auf die besonderen Anliegen der EFTA, des Commonwealth-Handels und der britischen Landwirtschaft vorgebracht worden war..$^{50}$

Aus all dem ergab sich die vollständige Übernahme von Pflichten aber auch aller Rechte zum Zeitpunkt des Beitritts, insbesondere in Bezug auf die Repräsentation und Mitwirkung der Neumitglieder in den Gemeinschaftsinstitutionen. Eine abgestufte Mitgliedschaft war ausgeschlossen, ebenso kam keine „Erweiterungsflexibilisierung“51 zum Zuge. So urteilt Gstöhl: „Mitgliedstaaten können bei Vertragsrevisionen oder im Rechtsetzungsverfahren Sonderwünsche durchsetzen, Beitrittsländer nicht. Ausnahmen, die die alten Mitgliedstaaten für sich beanspruchen, stehen den neuen Vertragsparteien in der Regel nicht zur Wahl, denn frühere Kompromisslösungen sollen nicht in Frage gestellt werden. "52 Vielfach waren gerade von akademischer Seite viel größere Erwartungen an die Binnendifferenzierung der EU im Zuge der Aufnahme neuer Mitglieder gerichtet worden. ${ }^{53}$ Andererseits herrschte bereits bei der Süderweiterung, also vor Schaffung des Binnenmarktes, diesbezüglich große Skepsis. Es stellte sich die Frage, ,ob im Rahmen einer Vollmitgliedschaft abgestufte Lösungen gefunden werden können. ... [D]aß der Spielraum dafür gering ist, weil gerade die weniger entwickelten Mitgliedstaaten aus politischen Gründen keine zweitklassige Rolle akzeptieren werden. Man könnte eine solche Lösung im übrigen auch nicht auf die Behandlung der Beitrittsländer beschränken, sondern müsste innerhalb der EG der Zwölf zwischen Kernländern und anderen Ländern unterscheiden; dies wäre aber sicher nicht durchzusetzen. Die Bildung eines solchen ,Gemeinschaftskerns “ würde auch voraussetzen, daß die beteiligten Länder bereit sind, sich einer stärkeren Gemeinschaftsdisziplin zu unterwerfen. “54 Der Spill-over

47 Vgl. Entwurf des Mandats für die Regierungskonferenz, in: Europäischer Rat: Schlussfolgerungen des Vorsitzes, 21./22. Juni 2007, 11177/07, Brüssel 2007, 23.6.2007, S. 27.

48 Vgl. das Kommuniqué der Staats- und Regierungschefs der Mitgliedstaaten der Europäischen Gemeinschaften in Den Haag am 1. und 2. Dezember 1969, abgedruckt in Nass: Englands Aufbruch, 1971, S. 99-103.

49 Abgedruckt in: Nass: Englands Aufbruch, 1971, S. 104-115, hier S. 110.

50 Vgl. die Bedingungen, die Macmillan 1961 stellte mit dem unkonditionierten zweiten Antrag der Regierung Wilson vom Mai 1967. Vgl. Nass: Englands Aufbruch, 1971, S. 13-15. Natürlich formulierten Kandidaten Voraussetzungen für ihren Beitritt; siehe beispielsweise Heinrich Schneider: Der österreichische Antrag auf EG-Mitgliedschaft: Der Kandidat stellt Bedingungen, in: integration 4/1989, S. 143-151. Schweden verhielt sich also mit seinem quasi Opt-out aus der WWU regelwidrig, was von der EU bis dato geduldet wird.

51 Sieglinde Gstöhl: Die Flexibilität der Europäischen Union gegenüber Mitgliedern und Drittstaaten, in: Jörg Glombowski u.a. (Hrsg.): Erweiterung und Integration der EU, Wiesbaden 2004, S. 147-173, hier S. 170.

52 Ebenda.

53 Vgl. statt vieler mit Blick auf den möglichen Beitritt von MOE-Ländern Anna Michalski/Helen Wallace: The European Community. The Challenge of Enlargement, London 1992. 
auf das EU-System und die als Risiko eingestufte Erosion des Gleichheits- und Uniformitätsgedankens wurde bereits für eine mit der Süderweiterung heterogener werdende Gemeinschaft kalkuliert. Eine weithin geteilte Schlussfolgerung war aber, dass nur pragmatische Lösungen und keine Patentlösungen aussichtsreich waren. ${ }^{55}$ Damit war - wie vor allem von Vertretern des (historischen) Institutionalismus und Neo-Funktionalismus betont ${ }^{56}-$ der $^{2}$ Pfad von begrenzten, fallbezogenen Anpassungsschritten eingeschlagen, der der EG - ganz im Sinne der „Methode Monnet“57 - eine ausreichende Beweglichkeit und Reaktionsmöglichkeit im Zuge der Erweiterung ließ. Demgegenüber hatte es immer auch Versuche gegeben, einen längerfristigen Orientierungsrahmen für die politische Entwicklung der Union im Zeichen des anhaltenden Erweiterungsdrucks und großer Beitrittserwartungen europäischer Staaten zu beschließen oder zumindest eine strukturierte Diskussion darüber auf die EU-Tagesordnung zu setzen. Die Initiative ergriff zumeist Frankreich, zuletzt mit dem Vorschlag von Präsident Sarkozy, ${ }^{58}$ einen „Rat der Weisen“ zu Zukunftsfragen der EU (wie sich Europa in den nächsten 30 Jahren! entwickeln soll) einzuberufen. Im Jahr 1999 hatte schon Jacques Attali für den französischen Außenminister einen Bericht „Europe 2020: Pour une Union plurielle“59 erarbeitet. In der Vergangenheit sind Versuche, eine ,grand débat“ zu entfachen allerdings im Sande verlaufen.

Festzustellen ist in unserem Zusammenhang: Die Erweiterungen haben zum weiteren Ausfransen und zur Komplexität des Gemeinschaftssystems beigetragen: durch Übergangsregelungen (etwa für die Gemeinsame Agrarpolitik, die Arbeitnehmerfreizügigkeit, Umwelt, den freien Warenverkehr, die Dienstleistungsfreiheit), durch neue Politikinstrumente, statt tiefgreifender Reform der bestehenden (Kohäsionsfonds, neue Ziele der Strukturfonds), und durch Zulauf von langsameren Kandidaten in die Nachzüglerkreise im Falle von Politiken, deren Ziele mit unterschiedlicher Geschwindigkeit und kriteriengesteuert (Eurozone, Schengen) verfolgt werden können.

Praktisch ergab sich zudem, dass die EG/EU mit mehreren Bewerbern bilateral aber parallel Verhandlungen führte und sie die Gleichzeitigkeit des Inkrafttretens aus Gründen der Anpassung der Verträge und der Ratifizierung anstrebte. Die Rekrutierung beziehungsweise der Zulauf von neuen Mitgliedern folgte der Logik geografischer Nähe und/oder enger Verflechtung unter den Kandidaten. Bisher blieb diesbezüglich Griechenland die Ausnahme, die nächste Ausnahme könnte Kroatien werden.

Ferner war die Selbstorganisation der EG-Seite und der Mitgliedstaaten entscheidend für die integrationsbestandswahrende Verhandlungsführung. Der Antrag des Vereinigten Königreichs von 1961 und die ersten Assoziierungsverhandlungen mit Griechenland und der Türkei konfrontierten die EG erstmals mit diesen Verfahrensfragen, die eine praktischer Ausfüllung der primärrechtlichen Verfahren bedeuteten und hochpolitisch waren. ${ }^{60}$ Vor allem das Tandem Rat-Kommission musste sein Zusammenspiel und die Rollenverteilung definieren.

54 Everling: Integrationspolitische Probleme, 1977, S. 84.

55 Ebenda, S. 86.

56 Vgl. Paul Pierson: The Path to European Integration: A Historical Institutionalist Analysis, in: Comparative Political Studies 2/1996, S. 123-163; Lippert : Glanzloser Arbeitserfolg, 2004, S. 17-19.

57 Vgl. den Beitrag von Wessels/Faber in diesem Heft.

58 Nicolas Sarkozy: Rede zur Eröffnung der 15. Botschafterkonferenz Paris, 27. August 2007, abrufbar unter: http://www.botschaft-frankreich.de/IMG/sarkozy_botschafterkonferenz.pdf (letzter Zugriff: 12.9.2007).

59 Vgl. Jacques Attali: Rapport au Ministre des Affaires Étrangères - Europe 2020: Pour une Union plurielle, Paris, 19. Juillet 1999, abrufbar unter: http://www.attali.com (letzter Zugriff: 05.09.2007); vgl. dazu und zur Integrationsfähigkeitsdebatte Barbara Lippert: Die Erweiterungspolitik der Europäischen Union, in: Werner Weidenfeld/Wolfgang Wessels (Hrsg.): Jahrbuch der Europäischen Integration 2007, Baden-Baden 2007 (im Erscheinen).

60 Vgl. in diesem Zusammenhang Ceylanoglu: Assoziierungsabkommen im Vergleich, 2004, S. 105-164. 
Mit den 1970 einsetzenden Verhandlungen wurde das nunmehr klassische Verfahren eingeführt: Der Rat und damit die EG, also nicht die Mitgliedstaaten, verhandeln mit dem Bewerber auf allen Ebenen und zwar auf der Grundlage einer gemeinsamen Position, die durch den Ratsvorsitz vertreten und im Rahmen der üblichen AStV-Prozeduren erarbeitet wird. Hingegen sollte beim ersten britischen Antrag 1961 ein gemeinsamer Standpunkt nur ,nach Möglichkeit“ zugrunde gelegt werden. Diese ersten Verhandlungen liefen noch zwischen den sechs EWG-Mitgliedstaaten und dem Vereinigten Königreich ab und jede Delegation hatte das Recht, das Wort zu ergreifen. Demgegenüber betonte der Ratsvorsitzende Harmel 1970: „Es geht also dieses Mal um Verhandlungen im Rahmen einer Konferenz der Gemeinschaften mit den Staaten, die einen Antrag ... gestellt haben. “61

Zwar schmetterte der Rat 1969 das Ansinnen der Kommission ab, für die Verhandlungen mit Großbritannien ein generelles Verhandlungsmandat vom Rat zu erhalten. Dennoch geriet die Kommission zunehmend in eine Schlüsselposition. Sie baute ihre Rolle vor allem im Zuge der Osterweiterung und der sie begleitenden Heranführungsstrategie noch einmal deutlich aus und etablierte dafür eine eigene Generaldirektion Erweiterung. Die Kommission spielte aber von Anbeginn neben der technischen auch eine politische Rolle im Erweiterungsprozess: Durch den Entwurf der Gemeinsamen Position des Rates bestimmte sie die Agenda. Vom Rat mandatiert, bei den Kandidaten Lösungen für spezifische Probleme zu sondieren, wurde sie zur zentralen Vermittlerin, wobei sie im Auge hatte, dass es bei der Lösungssuche nicht um „uns und jene“ sondern ,the future us “62 geht, und somit bereits um das Wohl der erweiterten Gemeinschaft. Ihr Vorteil war die Kombination aus Expertenwissen, institutionellem Gedächtnis und personeller Kontinuität vor allem gegenüber dem rotierenden Vorsitz des Rats.

Schon bei der Norderweiterung hatte die Kommission jene Problemfelder für die EG klar identifiziert, die bei jeder der nachfolgenden Erweiterungsrunden wieder diskutiert wurden: Institutionen, Haushalt und sektorale Sensibilitäten von Mitgliedstaaten, insbesondere bei finanzwirksamen Politiken wie der Gemeinsamen Agrarpolitik und später der Strukturpolitik. Eine Besonderheit blieben als Erbe des britischen Beitritts die langjährigen Nachverhandlungen über den „britischen Rabatt““63 Die Kommission betonte, wie oben dargelegt, seit dem ersten britischen Beitrittsantrag den Zusammenhang von Vertiefung und Erweiterung im Sinne eines parallelen Prozesses mit der Tendenz, den Vorrang bei der Vertiefung zu suchen. ${ }^{64}$ Das vertrat besonders prägnant Kommissionspräsident Delors und bekräftigte es mit seiner EWR-Initiative, dem einzigen effektiven Versuch, eine Zwischenlösung oder Alternative zur Erweiterung zu finden.

Überwölbt wurde das Tandem Rat-Kommission von den Staats- und Regierungschefs beziehungsweise seit seiner Gründung vom Europäischen Rat. Er fungiert gerade in Fragen der Erweiterung als Weichensteller für Entscheidungen von historischer Tragweite (polity), muss aber auch als Schlichter und Makler für viele Detailentscheidungen herhalten: Demnach gibt er grünes Licht für die Aufnahme und den Abschluss von Beitrittsverhandlungen so bei der Erweiterung 2004 - und ist bis in die Details mit dem Gesamtpaket für die Aufnahme neuer Mitglieder befasst, so beim Gipfel in Kopenhagen 2002. ${ }^{65}$ Zwar setzen Mit-

61 Zit. nach Nass: Englands Aufbruch, 1971, S. 114.

62 Graham Avery: The Commission's Perspective on the Negotiations, SEI Working Paper No. 12, 1995, S. 1.

63 Vgl. Brigid Laffan/Michael Shackleton: The Budget. Who Gets What, When, and How, in: Helen Wallace/William Wallace (Hrsg.): Policy-Making in the European Union, Oxford/New York 2000, S. 211-241, hier S. 217-226.

64 Vgl. Kommission der Europäischen Gemeinschaften: Stellungnahme zum griechischen Beitrittsgesuch, 29.1.1976, abgedruckt in: Hasenpflug/Kohler: Süd-Erweiterung, 1977, S. 227-268.

65 Vgl. Barbara Lippert: Von Kopenhagen bis Kopenhagen: Eine erste Bilanz der EU-Erweiterungspolitik, in: Aus Politik und Zeitgeschichte B1-2/2003, S. 7-15. 
gliedstaaten unabhängig von ihrer Größe ihre Präferenzen bei der Ausgestaltung der Beitrittsverträge (zum Beispiel im Hinblick auf Übergangsregelungen) und die damit verbundenen politisch-institutionell-finanziellen Beschlüssen durch. Doch sind es die groBen Mitgliedstaaten - vor allem Frankreich und Deutschland und teilweise das Vereinigte Königreich - unter denen eine Verständigung über Beginn und Abschluss der Verhandlungen und die großen Linien des Gesamtpakets gefunden werden muss.

Seit dem Vertrag von Maastricht muss zudem das Europäische Parlament jeder Erweiterung zustimmen. Es ist die einzige direkte Beteiligung des Europäischen Parlaments an einer Änderung des Primärrechts. Es begleitete alle Beitrittsverhandlungen intensiv, und betonte traditionell besonders die politische Dimension. Obwohl es seine Forderungen ${ }^{66}$ nach ,Vertiefung vor Erweiterung ' oftmals - wie im Falle der EFTA-Erweiterung 1995 - nicht durchsetzen konnte, blieb die Zustimmungsquote zur Aufnahme neuer Mitglieder mit 75 bis 92 Prozent stets sehr hoch. ${ }^{67}$ Das Europäische Parlament hat in keinem Fall von seiner Vetomöglichkeit Gebrauch gemacht.

Bisher verlief die Ratifikation von Beitrittsverträgen auf EG/EU Seite ohne Probleme. Zu den Besonderheiten zählte die Volksabstimmung in Frankreich über die Norderweiterung im Jahr 1972 und die potenzielle Superschutzklausel für den Aufschub des Beitritts von Rumänien und Bulgarien um ein Jahr. Vetos wurden nur zweimal von Seiten der Bevölkerung Norwegens eingelegt und damit der Beitritt des Landes zu Fall gebracht. Neuerdings sollen die Bürgerinnen und Bürger der Mitgliedstaaten als potenzielle Vetospieler stärker ins Spiel gebracht werden, wie es die neue Verfassungsbestimmung in Frankreich zu Erweiterungen ermöglicht.

Als Gatekeeper kann die EG/EU ihre Präferenzen und Konditionen durch die Festlegung des Termins für den Beginn und den Abschluss der Verhandlungen am stärksten geltend machen. Zwar hatte die Kommission schon im Falle Griechenlands (vergeblich) versucht, wenigstens den Vorlauf zur Antragsstellung zu strukturieren und stufenweise zu konditionieren. Aber erst die Osterweiterung brachte mit der Heranführungsstrategie eine echte Verfahrensinnovation. Die EU versucht im Rahmen der Heranführungsphase durch eine Politik der Konditionalität (Kopenhagener Kriterien) ,perfekte Mitglieder“ 68 zu erziehen. Sie macht bereits die Eröffnung von Verhandlungen von der völligen oder teilweisen Erfüllung der politischen und wirtschaftlichen Beitrittskriterien abhängig und verfolgt deren weitere Implementierung und Einhaltung durch ein aufwendiges Monitoringverfahren während der Verhandlungen bis zur Aufnahme. Steuerungsversuche wendet die EU gegenwärtig bei den Ländern des westlichen Balkans an, die einen klar definierten Stufenplan bis zur Eröffnung von Verhandlungen absolvieren sollen. ${ }^{69}$ Weitere Beispiele boten die Türkei und Kroatien. ${ }^{70}$

66 Vgl. Europäisches Parlament: Entschließung zur Gestaltung und Strategie der Europäischen Union im Hinblick auf ihre Erweiterung und die Schaffung einer gesamteuropäischen Ordnung, 20. Januar 1993, abrufbar unter: http://www.europarl.europa.eu/enlargement/positionep/resolutions/200193_de.htm (letzter Zugriff: 24.9.2007).

67 Vgl. im Einzelnen: Finnland - Zustimmung: 377, Ablehnung: 21, Enthaltung: 61; Norwegen: 376:24:57; Österreich: 378:24:60; Schweden: 381:21:60; Estland: 520:22:24; Lettland: 522:22:24; Litauen: 521:22:24; Malta: 521:23:23; Polen: 509:25:31; Slowakei: 521:22:22; Slowenien: 522:22:22; Tschechien: 489:39:37; Ungarn: 522:23:23; Zypern: 507:29:26; Bulgarien: 522:70:69; Rumänien: 497:93:71.

68 Alan Mayhew: Enlargement of the European Union: An Analysis of the Negotiations with the Central and Eastern European Candidate Countries, Sussex European Institute Working Paper No. 39, 2000, S. 10.

69 Michael Dauderstädt/Barbara Lippert/Andreas Maurer: Die deutsche EU-Ratspräsidentschaft 2007: Hohe Erwartungen bei engen Spielräumen, in: Friedrich-Ebert-Stiftung (Hrsg.): Internationale Politikanalyse, Bonn 2006, S. 30-31.

70 Vgl. zur Türkei Barbara Lippert: Die Türkei als Sonderfall und Wendepunkt der klassischen EU-Erweiterungspolitik, in: integration 2/2005, S. 119-135. 
Die EG war schon bei der Norderweiterung - eingedenk der Haager Beschlüsse - bestrebt, den Ausbau der Gemeinschaft parallel weiter zu verfolgen: Das heißt Beitrittsverhandlungen führen nicht zu einem Moratorium bei der, Vertiefung'. Bei der in Aussicht genommenen Norderweiterung wurden Anfang der 1970er Jahre die WWU, die Einrichtung des Europäischen Rates und der EPZ parallel verfolgt, parallel zur Süderweiterung wurden die Schaffung des Binnenmarktes, der WWU, der GASP sowie der Zusammenarbeit bei der Innen- und Justizpolitik in Angriff genommen. Es handelte sich aber weder um ein Junktim noch um eine strikte Sequenzierung von Vertiefung und Erweiterung. Nach allgemeiner Einschätzung und bekräftigt durch die Revisionskonferenz von Nizza und dem sogenannten Laeken-Prozess hat die Vertiefung seit Mitte der 1990er Jahre mit der Erweiterung nicht Schritt halten können. Die Doppelstrategie zur Vertiefung vor, nach oder parallel zur Erweiterung blieb die Achillesferse der Erweiterungsdoktrin. ${ }^{71}$

Die hier skizzierte Erweiterungsdoktrin ließ die Frage offen, ob es legitim sei, einem beitrittsreifen Bewerber die Aufnahme zu verweigern, zum Beispiel im Sinne eines pro-aktiven strategischen Verhaltens der EG/EU, wie es für einen Staat wie die USA (zum Beispiel gegenüber Mexiko) oder einen imperialen Akteur zu rechtfertigen wäre.

\section{Langzeitfolgen}

Mit Blick auf die Langzeitfolgen der Aufnahme neuer Mitglieder ragen die Nord- und die Osterweiterung heraus.

Dem Beitritt des Vereinigten Königreichs von 1973 kommt eine Schlüsselbedeutung für den Gang des Integrationsprozesses zu. Er hat das politische Kräfteverhältnis in der EG nachhaltig verändert und das neue Grundmuster westeuropäischer Politik „Paris - Bonn London“72 definiert. Das Leitbild des intergouvernementalen Europas erhielt mit dem Vereinigten Königreich einen starken Pol und setzte eine neue integrationspolitische Balance in der EG/EU in Gang. Als Ständiges Mitglied des UN-Sicherheitsrats und als Akteur mit imperialer Vergangenheit hat das Vereinigte Königreich der EG einen kräftigen Impuls für den Ausbau und die Profilierung ihrer außen- und sicherheitspolitischen Rolle gegeben. Es ist bis heute der einzige Kandidat, der auf Augenhöhe mit der EU verhandelt und um dessen Beitritt die EG nachdrücklich geworben hat. Zudem stärkte Britannien die wettbewerbsorientierten und freihändlerisch-liberalen Positionen in der EG, initiierte eine Dauerkritik an der Gemeinsamen Agrarpolitik und zwang die EG, sich mit den Ungereimtheiten des Eigenmittelsystems zu befassen. Der Beitritt des Vereinigten Königreichs wäre in seiner integrations- und geopolitischen Dimension nur von einem künftigen Beitritt der Türkei zu übertreffen, und zwar im Hinblick auf die Verschiebung der innerunionalen politischen Kräfteverhältnisse (zum Dreieck ,Paris-Berlin-London' würde Ankara hinzutreten), auf die geostrategische Ausrichtung der Union im Krisenbogen des Mittleren Ostens und in Hinblick auf die Tiefe des Reformbedarfs von Politiken, Haushalt und Institutionen der EU.

Die zweite Zäsur für die Integrationspolitik war das Epochenjahr 1989, mit dem die weltpolitischen Beschränkungen der EU wegfielen und sich die gesamteuropäische Ordnungsfrage mit einem Mal neu stellte. Darauf war die zentrale Antwort der EG/EU, sich zu erwei-

71 Barbara Lippert: Enlargement: the political and constitutional implications, in: Fergus Carr/Andrew Massey (Hrsg.): Public Policy and the new European Agendas, Cheltenham/Northampton 2006, S. 99-131; Anne Faber: Die Weiterentwicklung der Europäischen Union: Vertiefung versus Erweiterung?, in: integration 2/2007, S. 103-116.

72 Werner Link: Außen- und Deutschlandpolitik in der Ära Brandt 1969-1972, in: Karl Dietrich Bracher/Wolfgang Jäger/Werner Link (Hrsg.): Geschichte der Bundesrepublik Deutschland. Republik im Wandel 19691972. Die Ära Brandt, Stuttgart 1986, S. 163-282, hier S. 242. 
tern. Damit kam der EU-Erweiterung erstmals eine geostrategische Bedeutung zu: Aus deutscher Sicht war das ansatzweise bereits 1973 der Fall, als die Erweiterung als eine Voraussetzung für die europäische Einbettung der deutschen Ostpolitik im Sinne ,gesamteuropäischer Ordnungspolitik “73 angesehen wurde. Bundeskanzler Brandt sagte auf dem Haager Gipfel 1969 „,daß unsere Gemeinschaft kein neuer Block, sondern eine exemplarische Ordnung sein soll, die als Bauelement einer ausgewogenen gesamteuropäischen Friedensordnung taugt" ${ }^{74}$ Zwei Aspekte sind hier festzuhalten: zum Einen wird die EG als Modell und nicht als klassische Großmacht dargestellt und auf ihre magnetische Wirkung vertraut; zum Anderen wird sie als eigener Faktor bei der Gestaltung Gesamteuropas angesehen, wie es die EG mit Hilfe der sich entwickelnden EPZ im Rahmen des Helsinki/KSZE-Prozesses versuchte. ${ }^{75}$ Aber überwiegend wurde in den 1970er Jahren die strukturbildende Bedeutung der ersten Erweiterung noch als auf das Wirtschaftliche beschränkt angesehen: „Consequently, the prospects of enlargement bring the Community face to face with the problem of the economic organisation of a large part of Europe...." ${ }^{" 76}$ Erst die Erweiterung von 2004 vollzieht den Paradigmenwechsel zur geostrategischen Begründung und Bedeutung der Erweiterung, allerdings unter Beibehaltung der überkommenen integrationspolitischen Ziele des wirtschaftlichen Wachstums und sozialen Zusammenhalts sowie der Gleichheit und Solidarität im Inneren. Die Spannung zwischen diesen außen- und binnengeleiteten Zielen bestimmt gegenwärtig die Debatte über die Integrations- und Absorptionsfähigkeit der EU.

\section{Resümee und Ausblick}

Welche Lehren für die Zukunft lassen sich ableiten? Die EU kann und will das Kapitel „Erweiterung“ auf absehbare Zeit nicht schließen. Das Feld potenzieller Kandidaten hat sich schon deutlich formiert: Den engsten Kreis bilden neben den verbliebenen EFTA-Ländern die Türkei und Kroatien, mit denen Verhandlungen laufen, und ferner die bereits mit einer europäischen Perspektive vorgemerkten Kandidaten des westlichen Balkans. Die EU hat gegenüber diesen Ländern alle Signale auf Aufnahme gestellt. Sie kann deren Zulauf zu steuern versuchen, indem sie den Zeitpunkt der Eröffnung und des Abschluss von Verhandlungen bestimmt sowie maßgeblich die genauen Bedingungen des Beitritts festlegt. Bei den übrigen Ländern Osteuropas, von denen bereits die Ukraine, Moldova und teilweise Georgien, ein Beitrittsinteresse bekundet haben, stellt sich zunächst die Frage, inwieweit die Europäische Nachbarschaftspolitik durch eine wirksame Strategie der Modernisierung und politischen Einbindung den Erweiterungsdruck mindern kann. Solange sich auf dem Kontinent kein demokratisches und prosperierendes subregionales Zentrum neben der EU etabliert, bleibt sie der Fixpunkt für alle europäischen Staaten mit der wahrscheinlichen Ausnahme von Russland. Die eigentliche Dynamik der Erweiterung geht auf Dauer von der Türkei und den defekten oder höchstens teildemokratisierten Staaten des Ostens aus, für die eine schwache Staatsführung in Kombination mit wirtschaftlicher und sozialer Rückständigkeit kennzeichnend sind. Sie erwarten von dem Beitritt zur EU eine Catch-all-Lösung für ihre gravierenden strukturellen Probleme und Defizite in allen drei Sachbereichen: Wohlfahrt,

73 Werner Link: Außen- und Deutschlandpolitik in der Ära Schmidt, in: Wolfgang Jäger/Werner Link (Hrsg.): Republik im Wandel 1974-1982. Die Ära Schmidt, Stuttgart 1987, S. 273-446, hier S. 428.

74 Erklärung des Bundeskanzlers Willy Brandt auf der EWG-Gipfelkonferenz in Den Haag, in: Bulletin des Presse- und Informationsamtes der Bundesregierung vom 2.12.1969, Nr. 146, S. 1241.

75 Vgl. Heinrich Schneider: The Twelve/Fifteen's Conference Diplomacy: Has the CSCE/OSCE Remained a Successful Platform?, in: Elfriede Regelsberger/Philippe de Schoutheete de Tervarent/Wolfgang Wessels (Hrsg.): Foreign Policy of the European Union. From EPC to CFSP and Beyond, London 1997, S. 237-261.

76 Commission: Opinion, 1969, S. 25. 
Sicherheit und Herrschaft. Der EU fehlt zwar noch ein eng an die eigene politische Identität gekoppeltes Narrativ, mit dem sich politische Unterstützung für die Aufnahme der osteuropäischen Länder innerhalb der EU mobilisieren ließe. Dafür könnte sie an die politisch identitären Motive, die die Osterweiterung getragen haben (Stabilitätsexport, Demokratiehelfer von außen), anknüpfen, die gerade im Falle einer zugespitzten außen- und sicherheitspolitische Lage der Kandidaten , abrufbar' würden. Das Lager der Erweiterungsunterstützer hat durch die zwölf neuen Mitgliedstaaten weiteren Zulauf erhalten. In Deutschland, einem Schlüsselstaat für die Erweiterungsgeschichte 1973-2007, wird jedoch die Position zur Erweiterung in den politischen Parteien und der Bevölkerung kritischer. Die Bundesregierung trat in der Vergangenheit nicht nur als Befürworter, sondern häufig auch als Konziliator auf, der Politik- und finanzielle Angebote einbrachte, um Kompromisse in Beitrittsverhandlungen zu erzielen und der Aufnahme neuer Mitglieder zum Durchbruch zu verhelfen. ${ }^{77}$ Als einer der Gatekeeper für die Aufnahme und den Abschluss von Beitrittsverhandlungen suchte sie immer das Zusammenwirken mit Frankreich, auf dessen skeptischere Position sich Deutschland bislang nur in der Frage des Türkei-Beitritts zu bewegt, sich jedoch beispielsweise in Bezug auf die europäische Perspektive der Ukraine offener zeigt. Die Präferenzbildung in Deutschland zwischen Konsolidierung und weiterer Vertiefung auf der einen und Erweiterung auf der anderen Seite könnte für die künftige Dynamik der EU-Erweiterung entscheidend werden.

Davon abgesehen verstärken auch endogene Entwicklungen in der EU die Erweiterungsdynamik: Aufgrund ihrer als modellhaft geltenden, erfolgreichen Heranführungsstrategie und Politik der Konditionalität gegenüber den Beitrittsländern von 2004/07 schafft die EU einen starken Anreiz für die postsowjetischen Transformationsländer Osteuropas. Die EU hat zudem auch nach der Erweiterung 2004/07 ihre Politik der Offenheit gegenüber allen europäischen Staaten und ihr Selbstverständnis als politisch unabgeschlossenes Projekt keineswegs revidiert, sondern sogar im Zuge des Verfassungsprozesses rhetorisch verstärkt. ${ }^{78}$ Als nicht-imperialer Akteur vermag die EG/EU keine Expansionspolitik und pro-aktive Erweiterungspolitik zu betreiben, sondern reagiert auf Beitrittsbegehren und Forderungen der AuBenwelt.

Aufs Ganze betrachtet zeigen sich in der Geschichte der Erweiterung Motive, Erwartungen und Strategien seitens der EG/EU-Akteure, die von Runde zu Runde auf neuem Niveau der Integration wiederkehrten. Seit der ersten Erweiterung sind Sorgen vor einer institutionellen, finanziellen und nicht zuletzt politischen Überdehnung und dem damit verbundenen identitären Wandel anzutreffen. Demgegenüber stand jedes Mal die positive Erwartung, dass die Gemeinschaft im Zuge der Erweiterung an wirtschaftlicher Macht und politischem Einfluss gewinnen und dass die Erweiterung als Schwungrad für die weitere Verfolgung jeweiliger europapolitischer Strategien zu nutzen sein wird. So war die Erweiterung für Anhänger supranational-gemeinschaftlicher (das Europäische Parlament, die Kommission und integrationsfreundliche Mitgliedstaaten) Entwicklungsperspektiven ebenso akzeptabel wie für souveränitätsorientierte Mitgliedstaaten. Die Entwicklungsoffenheit der EG/EU diskreditierte geradezu eine ablehnende Position gegenüber dem Begehren von beitrittsfähigen europäischen Staaten. Bei skeptischen Mitgliedstaaten paarte sich resignative Zustimmung

77 Vgl. Barbara Lippert: Die EU-Erweiterungspolitik nach 1989 - Konzeptionen und Praxis der Regierungen Kohl und Schröder, in: Heinrich Schneider/Mathias Jopp/Uwe Schmalz (Hrsg.): Eine neue deutsche Europapolitik?, Bonn 2001, S. 349-392, besonders S. 362.

78 Vgl. Barbara Lippert: Die Erweiterungspolitik der Europäischen Union, in: Werner Weidenfeld/Wolfgang Wessels (Hrsg.): Jahrbuch der Europäischen Integration 2006, Baden-Baden 2007, S. 429-452. 
zum als unabänderlich Angesehenen mit der Erfahrung, dass sich negative AbschreckungsSzenarien in der Realität nicht oder nur sehr abgeschwächt durchgesetzt hatten.

Denn das System der EG/EU erwies sich über die Erweiterungsrunden hinweg als stabil und dauerhaft. Eine Schlüsselbedeutung kommt der Erweiterungsdoktrin zu, die in den Kernpunkten seit der ersten Norderweiterung fest etabliert und danach ausdifferenziert worden war. Das EU-System hat eine hohe Anpassungsfähigkeit bewiesen, was wiederum negativen Prognosen (Paralyse des Entscheidungssystems, Zusammenbruch des Haushalts etc.) die Plausibilität nahm. Durch das Festhalten am Acquis als Eintrittsbedingung für Neumitglieder baute die EU einen Schutzwall gegen die Erosion des EU-Systems auf. Wer die Axt an diesen Grundsatz legt und etwa - wie im Verhandlungsrahmen für die Türkei und Kroatien angedeutet - dauerhafte Ausnahmen und eine Mitgliedschaft zweiter Klasse oder eingeschränkter Art beschließt, leitet eine Wende der Erweiterungspolitik und ihrer Wirkungen auf das EU-System ein. ${ }^{79}$ Zwar ist auch schon bei früheren Erweiterungsrunden das Ende der klassischen Erweiterungspolitik verkündet oder erwartet worden, ${ }^{80}$ doch sind neuere Tendenzen in diese Richtung zu erkennen. ${ }^{81}$

Unter den Faktoren, die den Ausbau und die Differenzierung des politischen Systems der EU und seiner Politiken bestimmten, wurde stets auf die Aufnahme neuer Mitglieder und den dadurch hervorgerufenen Reformdruck hingewiesen. Es ist methodisch und empirisch ausgesprochen schwierig, die erweiterungsinduzierten Wirkungszusammenhänge und damit die politisch-konstitutionellen Implikationen der Erweiterung gegenüber anderen Einflüssen zu isolieren. Wie groß auch immer die Bedeutung der Erweiterung für die Vertiefung in der Vergangenheit zu veranschlagen war, als Quelle für Veränderungen hat sie allein nie ausgereicht. Das in allen Erweiterungsrunden bemühte Argument des Schritthaltenmüssens mit der Vergrößerung der Gemeinschaft durch rechtzeitige vertikale Vertiefung überzeugte sogar immer weniger. So wird die EU mindestens bis 2009 mit dem als unzureichend angesehenen Vertragssystem von Nizza arbeiten.

Geht man davon aus, dass selbst die bis dato größte Erweiterung in der Geschichte der EU (2004/07), die noch dazu von epochaler politischer Bedeutung war, kein ausreichendes Momentum für einen qualitativen Sprung der Gemeinschaftsentwicklung erbrachte, dann lässt sich dies auch als wahrscheinliches Szenario für die Zukunft annehmen. Die Erweiterung hat als rhetorisch genutztes Schwungrad oder als effektiver Motor der Vertiefung im Sinne eines Ausbaus gemeinschaftlicher Verfahren, Politiken und Ressourcen ausgedient. Für über begrenzte inkrementelle Anpassungen hinausgehende Entwicklungsschübe des EU-Systems, wird die EU stärker auf andere Quellen der Legitimation als auf die Erweiterung zurückgreifen oder dazu von externen Schocks genötigt werden müssen. Es dürfte jedoch nicht einfach sein, ein neues großes Projekt zu finden, das wie die Erweiterung alle drei Dimensionen der polity, der politics und der policy umfasst und damit große Möglichkeiten bietet, auch widerstreitende Präferenzen und Strategien zu bündeln. In dieser Projektionsmöglichkeit lag lange Zeit der strategische Reiz und Vorteil der Erweiterungspolitik. Während die Dynamik der Erweiterung mit verlangsamtem Tempo anhalten wird, ist die Anpassungsfähigkeit und Stabilität des EU-Systems ungewiss.

79 Lippert: Türkei als Sonderfall, 2005.

80 John Redmond/Glenda G. Rosenthal: Introduction, in: John Redmond/Glenda G. Rosenthal (Hrsg.): The Expanding European Union. Past, Present, Future, London 1998, S. 1-14, hier S. 4.

81 Vgl. Kai-Olaf Lang/Daniela Schwarzer: Argumente für eine neue Erweiterungsstrategie - die Diskussion über die Aufnahmefähigkeit der EU, in: integration 2/2007, S. 117-128. 